\title{
Organização da demanda em saúde bucal e a vulnerabilidade familiar
}

\author{
Organization of demand in oral health and family vulnerability
}

João Peres Neto (https://orcid.org/0000-0002-6988-5826) ${ }^{1}$

Karine Laura Cortellazzi (https://orcid.org/0000-0001-9584-9477) ${ }^{2}$

Maria da Luz Rosário de Sousa (https://orcid.org/0000-0002-0346-5060) ${ }^{2}$

${ }^{1}$ Hospital Israelita Albert Einstein. Av. Brigadeiro Faria Lima 1.188, Jardim Paulistano. 01451-001 São Paulo SP Brasil. joao.peres@einstein.br ${ }^{2}$ Faculdade de Odontologia de Piracicaba, Universidade Estadual de Campinas. Campinas SP Brasil.

\begin{abstract}
The scope of this study was to analyze the relationship of an instrument of family vulnerability with sociodemographic, dental, and spatial distribution in a given territory. The dependent variable was the classification of family vulnerability, proposed by Coelho and Savassi. The independent variables were selected on the basis of the theoretical model proposed by Andersen. Hierarchical multiple logistic regression models were estimated. Kernel density estimation was used for spatial analysis. Subjects who lived with more than 4 people $(O R=3.46$; 95\% CI), who were dissatisfied with their oral health $(O R=2.38 ; 95 \% C I)$, who were ashamed to smile and talk $(O R=3.03$, 95\% CI) were more likely to be "at risk" in the family. Spatial analysis enabled the visualization of an area of higher concentration of families "at risk". The relationship of the instrument of vulnerability analyzed with sociodemographic and dental factors, as well as the assistance in the visualization and identification of more vulnerable areas, are useful in the knowledge of the territory for the planning of actions in oral health, and the conclusion drawn is that the tool can be adopted for more equitable access by the oral health teams. Key words Risk factors, Self-assessment, Oral health, Spatial analysis
\end{abstract}

Resumo O objetivo deste estudo foi analisar a relação de um instrumento de vulnerabilidade familiar com fatores sociodemográficos e odontológicos e sua distribuição espacial em determinado território. A variável dependente foi a classificação de vulnerabilidade familiar, proposta por Coelho e Savassi. As variáveis independentes foram selecionadas baseadas no modelo teórico proposto por Andersen. Foram estimados modelos de regressão logística múltipla hierarquizada. O estimador de densidade de Kernel foi utilizado para análise espacial. Indivíduos que residiam com mais de quatro pessoas (OR = 3,46; IC 95\%), que estavam insatisfeitos com a saúde bucal ( $O R=2,38$; IC 95\%) e que tinham vergonha ao sorrir e falar $(O R=3,03$; IC 95\%) apresentavam mais chances de estar "em risco" familiar. A análise espacial possibilitou a visualização de uma área de maior concentração de famílias "em risco". A relação do instrumento de vulnerabilidade analisado com fatores sociodemográficos e odontológicos, além do auxílio na visualização e identificação de áreas mais vulneráveis, auxilia no conhecimento do território para o planejamento das ações em saúde bucal, de modo que concluímos que tal instrumento pode ser adotado para um acesso mais equânime por parte das equipes de saúde bucal.

Palavras-chave Fatores de risco, Autoavaliação, Saúde bucal, Análise espacial 


\section{Introdução}

As Equipes de Saúde Bucal (ESB), ao terem a possibilidade de inserção na Estratégia Saúde da Família (ESF), por meio da Portaria-MS no 1.444 , de 28 de dezembro de 2000, que cria o incentivo financeiro e define seu modo de trabalho voltado para a reorganização do modelo e a ampliação do acesso, se fortalece ainda mais com a publicação das diretrizes da Política Nacional de Saúde Bucal (PNSB) em 2004 pelo Ministério da Saúde (MS). O principal objetivo da PNSB foi a reorganização da saúde bucal por intermédio de uma reorientação do modelo, tendo como um de seus pressupostos que as ações sejam precedidas de um diagnóstico das condições de saúde- doença das populações por meio da abordagem familiar e das relações que se estabelecem no território onde se desenvolvem as práticas de saúde ${ }^{1,2}$.

Tal reorientação do modelo, pautada nas diretrizes propostas pelo MS, tem se mostrado mais efetiva na ampliação do acesso e da utilização dos serviços ${ }^{3}$. Entretanto, para que o acesso contemple as necessidades principais e prioritárias em saúde de uma população é importante saber se a utilização dos serviços resulta de uma interação de fatores, entre eles as necessidades e as percepções da saúde sob a ótica do usuário, que é determinada por uma necessidade percebida decorrente de sua situação de saúde e de seu conhecimento prévio da doença, sendo esta influenciada por fatores sociodemográficos, considerados um importante indicador de acesso ${ }^{4-7}$. A saúde bucal muitas vezes utiliza apenas indicadores clínicos nas análises realizadas, o que pode implicar certas limitações pelo fato de não conseguirem identificar a ocorrência de sintomas percebidos e relatados pelos usuários, como dor e desconforto, não abordando o impacto da morbidade no bem-estar do paciente. Além disso, não identificam os indivíduos doentes que procuram o serviço, tendo em vista que essa demanda é modulada por fatores econômicos, sociais e culturais. Como alternativa de ampliação das informações a serem analisadas, questionários que medem em que grau as doenças influenciam a vida das pessoas e as situações do contexto sociodemográfico podem contribuir para a busca de informações complementares ${ }^{8}$.

Em um olhar contextualizado, e tendo a família como foco do cuidado enquanto unidade de atuação da ESF, a possibilidade de utilização de um instrumento de estratificação de vulnerabilidade/risco familiar aplicado às famílias adscritas a uma equipe de saúde - a fim de determinar seu risco social e de saúde, refletindo o potencial de adoecimento de cada núcleo familiar, como é a escala de Coelho e Savassi - mostra-se útil no acesso e reorganização da demanda, promovendo uma percepção mais apurada, objetiva e qualificada do risco das famílias avaliadas, o que impacta de maneira positiva o trabalho da equipe $e^{9-12}$. Dessa maneira, um sistema de estratificação de vulnerabilidade/risco familiar que contemple aspectos epidemiológicos, sanitários e sociais da saúde pode ajudar a organizar o acesso em uma Unidade de Saúde da Família, permitindo identificar os grupos mais vulneráveis ${ }^{13}$.

Essa identificação dos grupos mais vulneráveis passa pelo conhecimento geográfico do território de atuação da equipe de saúde, e constitui uma das estratégias utilizadas para o diagnóstico e o planejamento das ações da ESF, que tem como um de seus princípios a adscrição da clientela em uma base territorial ${ }^{14}$. Os Sistemas de Informação Geográfica (SIG) possuem a capacidade de gerenciar dados pautados no componente geográfico do território, permitindo captura, armazenamento, manipulação, análise, demonstração e relatos de dados referenciados geograficamente. Isso permite que tais sistemas possam ser ferramentas apropriadas para auxiliar no processo de territorialização nos municípios ${ }^{15}$. As técnicas de geoprocessamento utilizadas pelos SIG vêm sendo utilizadas no planejamento, no monitoramento e na avaliação das ações de saúde, além de serem consideradas ferramentas importantes de análise das relações entre o ambiente e os eventos relacionados à saúde ${ }^{16}$.

Dessa maneira, o objetivo da presente pesquisa foi analisar a relação entre um instrumento de vulnerabilidade/risco familiar e fatores sociodemográficos e odontológicos e sua distribuição espacial em determinado território.

\section{Métodos}

Trata-se de um estudo transversal, no período de janeiro a junho de 2017, no município de Ubirajara, São Paulo, Brasil.

A amostra do trabalho foi o mesmo grupo de famílias participantes de um estudo inicial (Peres Neto e colaboradores, 2017) ${ }^{17}$, obtida por meio de um censo, e portanto representativa da população local, que foram classificadas segundo a vulnerabilidade/risco familiar proposta por Coelho e Savassi ${ }^{18}$. Foram incluídas todas as famílias do grupo inicial e excluídas as que não autorizaram o Termo de Consentimento Livre e Escla- 
recido (TCLE), as que mudaram de município e as que demandaram mais do que três visitas na residência para a coleta de dados.

As classificações de vulnerabilidade/risco das famílias foram reavaliadas pelo pesquisador principal, em virtude da dinamicidade com que as mesmas poderiam sofrer alterações, uma vez que as informações ou "sentinelas de risco" utilizadas possibilitam tais alterações.

Considerou-se como variável dependente a vulnerabilidade/risco familiar (Quadro 1), classificada segundo a escala de Coelho e Savassi ${ }^{18}$. A variável foi dicotomizada em "sem risco" (SR: sem risco = escore menor que 5) e "em risco" (R1: menor risco $=$ escore 5 ou 6; R2: médio risco $=$ escore 7 ou 8; e R3: máximo risc $\mathrm{o}=$ escore maior que 9).

Para a obtenção das variáveis independentes, foram aplicados pelo pesquisador principal, em visitas às residências, o mesmo questionário utilizado pelo Levantamento de Saúde Bucal do Estado de São Paulo 2015 (SB SP2015) $^{19}$, sendo selecionadas as variáveis que, baseadas no mode-

Quadro 1. Escala de risco familiar de Coelho e Savassi.

\begin{tabular}{|c|c|c|}
\hline \multicolumn{2}{|c|}{ Sentinelas de risco ${ }^{*}$} & \begin{tabular}{|c|} 
Escore \\
correspondente $^{\star}$
\end{tabular} \\
\hline \multicolumn{2}{|l|}{ Acamado } & 3 \\
\hline \multicolumn{2}{|c|}{ Deficiência física } & 3 \\
\hline \multicolumn{2}{|c|}{ Deficiência mental } & 3 \\
\hline \multicolumn{2}{|c|}{ Baixas condições de saneamento } & 3 \\
\hline \multicolumn{2}{|c|}{ Desnutrição grave } & 3 \\
\hline \multicolumn{2}{|c|}{ Drogadição } & 2 \\
\hline \multicolumn{2}{|c|}{ Desemprego } & 2 \\
\hline \multicolumn{2}{|c|}{ Analfabetismo } & 1 \\
\hline \multicolumn{2}{|c|}{ Menor de seis meses } & 1 \\
\hline \multicolumn{2}{|c|}{ Maior de 70 anos } & 1 \\
\hline \multicolumn{2}{|c|}{ Hipertensão arterial sistêmica } & 1 \\
\hline \multicolumn{2}{|c|}{ Diabetes mellitus } & 1 \\
\hline \multirow{3}{*}{$\begin{array}{l}\text { Relação } \\
\text { morador/ } \\
\text { cômodo }\end{array}$} & Se maior que 1 & 3 \\
\hline & Se igual a 1 & 2 \\
\hline & Se menor que 1 & 0 \\
\hline \multicolumn{3}{|l|}{ Total } \\
\hline \multicolumn{3}{|c|}{ Classificação de risco } \\
\hline \multicolumn{2}{|c|}{ Escore menor que 5} & SR - Sem Risco \\
\hline \multicolumn{2}{|c|}{ Escore 5 ou 6} & R1 - Menor \\
\hline \multicolumn{2}{|c|}{ Escore 7 ou 8} & R2 - Médio \\
\hline \multicolumn{2}{|c|}{ Maior que 9} & R3 - Máximo \\
\hline
\end{tabular}

${ }^{*}$ Valor de sentinela aplicada

Fonte: Menezes AHR, Cardelli AAM, Vieira GB, Martins JT, Fernandes MV, Marrero T-L18. lo teórico proposto por Andersen, determinam o acesso e a utilização dos serviços ${ }^{20}$. Seguindo esse modelo, as variáveis foram agrupadas em três blocos hierárquicos e organizadas, em relação ao desfecho, conforme indicado a seguir.

Bloco I - fatores predisponentes: idade (dicotomizada pela mediana em até 36 anos e acima de 36 anos), sexo (masculino e feminino), escolaridade (dicotomizada pela mediana em até nove anos e mais de nove anos de estudo), número de bens (dicotomizada pela mediana em até oito bens e mais de oito bens) e número de residentes no domićlio (dicotomizada pela mediana em até quatro moradores e mais de quatro moradores).

Bloco II - fatores facilitadores: renda familiar (dicotomizada pela mediana em até $\mathrm{R} \$ 500,00 \mathrm{e}$ mais de $\mathrm{R} \$ 500,00)$ e serviço de referência (dicotomizada em público e privado + convênios + outros).

Bloco III - necessidade percebida: motivo da consulta (dicotomizada em revisão + prevenção e dor + extração + tratamentos + outros), satisfação com saúde bucal (dicotomizada em satisfeito e insatisfeito), vergonha ao sorrir (dicotomizada em sim e não) e deixar de dormir (dicotomizada em sim e não).

A distribuição hierárquica das variáveis em cada bloco se iniciou pelos fatores predisponentes, seguindo até aquelas com maior proximidade da variável de desfecho.

Inicialmente foi realizada a análise descritiva dos dados. A seguir, uma análise bivariada para testar a relação entre as variáveis independentes e a dependente por meio da odds ratio (OR) e intervalos de confiança (IC) de $95 \%$.

Posteriormente foram estimados modelos de regressão logística múltipla hierarquizada pelo procedimento PROC GENMOD, considerando a distribuição binomial e a função de ligação logística. As variáveis que apresentaram $\mathrm{p} \leq 0,20$ foram testadas nos modelos de regressão logística múltipla, hierarquicamente, com início pelas variáveis do bloco I. Na sequência foram ajustados modelos com as variáveis do bloco II, e então com as variáveis do bloco III. Os ajustes foram avaliados pelo Akaike's information criterion (AICC).

As OR e os respectivos intervalos de confiança (IC) de 95\% foram estimados, e as variáveis tiveram como critério de permanência no modelo final $\mathrm{p} \leq 0,05$. Todas as análises foram realizadas no programa estatístico SAS $2010^{21}$.

A análise espacial do estudo foi realizada a partir do local das residências das famílias. Os endereços foram geocodificados por intermédio de um sistema de endereçamento universal 
("What3words.com") 22, que permitiu localizar todas as residências por meio de suas longitudes e latitudess $(\mathrm{X}, \mathrm{Y})$ e relacioná-las com a base cartográfica digital do município de Ubirajara/SP.

Utilizou- se o software QGIS 2.16.3 na criação da camada de pontos a partir das coordenadas $(\mathrm{X}, \mathrm{Y})$ obtidas na geocodificação para as análises e a elaboração dos mapas temáticos por meio do estimador de densidade de Kernel, que é um método não paramétrico para estimação de curvas de densidades em que cada observação é ponderada pela distância em relação a um valor central, o núcleo. $\mathrm{O}$ grau de alisamento utilizou um raio adaptativo, variando de acordo com a densidade dos pontos.

Esse estimador realiza uma contagem de todos os pontos dentro de uma região de influência, ponderando-os pela distância de cada um em relação à localização de interesse, facilitando a visualização de aglomerados, com a criação de mapas com níveis de densidades que variam de acordo com a cor e a tonalidade, representados da seguinte maneira: as classes mais escuras significam densidade muito alta; as com tonalidade média representam densidade média; e as com tonalidade mais clara indicam densidade baixa ${ }^{23}$.

Isso auxilia na análise porque quando uma faixa com uma tonalidade ou cor mais intensa é visualmente detectada, pode-se inferir que nesta região existe uma concentração elevada. Da mesma forma, quanto mais clara a cor, menos concentrada é a região.

A pesquisa foi aprovada pelo Comitê de Ética em Pesquisa da Faculdade de Odontologia de Piracicaba.

\section{Resultados}

Das 128 famílias possíveis da amostra do estudo inicial, foram efetivamente analisadas 115 famílias $(\mathrm{n}=115)$, representando uma perda amostral de $10,16 \%$ por mudança de município. Estavam "sem risco" 66 famílias (57,4\%), enquanto 49 famílias (42,6\%) estavam "em risco". $\mathrm{Na}$ reavaliação da classificação do risco familiar, não houve alteração nas mesmas desde o estudo inicial, realizado em 2015. A faixa etária variou de 21 a 67 anos, sendo que os valores da média e da mediana foram de $37,8(\mathrm{DP}=8,99)$ e 36 anos, respectivamente. A maioria dos participantes (87\%) que responderam como responsáveis pelas famílias era do sexo feminino.

$\mathrm{Na}$ análise bivariada (Tabela 1), o risco familiar se associou ao menor número de anos de estudo (OR = 2,424; IC 95\%: 1,131-5,193), à quantidade acima de quatro pessoas residentes no lar da família (OR = 3,255; IC 95\%: 1,471-7,202), à menor renda familiar $(\mathrm{OR}=2,537$; IC $95 \%$ : 1,049-6,132), à insatisfação com a saúde bucal $(\mathrm{OR}=3,189$; IC 95\%: 1,456-6,986), à vergonha ao sorrir (OR $=4,086$; IC 95\%: 1,526-10,943) e a deixar de dormir $(\mathrm{OR}=2,900$; IC 95\%: 1,105$7,609)$.

Nas tabelas 2 e 3 foram estimados os modelos de regressão logística múltipla hierarquizada para analisar a influência das variáveis independentes sobre o risco familiar. Após o ajuste para os três níveis hierárquicos, as variáveis que permaneceram no modelo final (Modelo 4) foram: número de residentes na casa, satisfação com a saúde bucal e vergonha ao sorrir. Sendo que os indivíduos que residem com mais de quatro pessoas na casa (OR = 3,4609; IC 95\%: 4665-8,1676), que estão insatisfeitos com a saúde bucal $(\mathrm{OR}=2,3858$; IC 95\%: $1,0039-5,6701)$ e que têm vergonha ao sorrir (OR = 3,0375; IC 95\%: 1,0102-9,1335) têm mais chances de estar em risco familiar.

A abordagem espacial do risco familiar é apresentada na Figura 1, no mapa de Kernel, que permitiu a identificação de uma área mais periférica da cidade com alta concentração de famílias "em risco" e outras duas com menor concentração.

\section{Discussão}

Segundo Bradshaw ${ }^{24}$, a necessidade normativa de tratamento, definida como aquela em que o profissional se baseia em padrões clínicos preestabelecidos para aplicá-lo, é a mais utilizada em estudos epidemiológicos. Todavia, muitas vezes demanda recursos materiais, humanos e financeiros, que frequentemente são limitados ${ }^{25,26}$. Como mecanismo alternativo e complementar, os questionários e as entrevistas são meios mais econômicos e convenientes de obter informações de saúde em primeira mão, por meio da autopercepção ou da necessidade relatada de tratamento. Tal percepção também pode ser considerada um indicador subjetivo da condição de saúde bucal, que está fortemente associado ao padrão de procura pelos serviços odontológicos e pode ser utilizado na definição de prioridades para o planejamento, visando a redução nas iniquidades ${ }^{27-32}$.

A autoprecepção em saúde bucal, validada enquanto instrumento de necessidade de tratamento odontológico em adultos, quando analisada com a necessidade normativa, permite sua utilização para fins de planejamento dos serviços, 
Tabela 1. Análise bivariada das variáveis independentes associadas ao risco familiar. Ubirajara, São Paulo, 2017.

\begin{tabular}{|c|c|c|c|c|c|c|c|c|}
\hline & & \multicolumn{4}{|c|}{ Risco Familiar } & \multirow{3}{*}{ OR } & \multirow{3}{*}{ IC 95\% } & \multirow{3}{*}{ p-valor } \\
\hline & & \multicolumn{2}{|c|}{ Sem risto } & \multicolumn{2}{|c|}{ Em risco } & & & \\
\hline & & $\mathbf{n}$ & $\%$ & $\mathbf{n}$ & $\%$ & & & \\
\hline \multirow{3}{*}{$\begin{array}{l}\text { Fatores } \\
\text { predisponentes }\end{array}$} & Idade & & & & & & & \\
\hline & Até 36 anos & 35 & 60,34 & 23 & 39,66 & Ref. & & \\
\hline & Acima de 36 anos & 31 & 54,39 & 26 & 45,61 & 1,276 & $0,609-2,677$ & 0,5185 \\
\hline \multirow[t]{12}{*}{ Bloco I } & Sexo & & & & & & & \\
\hline & Masculino & 10 & 66,67 & 5 & 33,33 & Ref. & & \\
\hline & Feminino & 56 & 56,00 & 44 & 44,00 & 1,571 & $0,501-4,932$ & 0,4387 \\
\hline & Escolaridade & & & & & & & \\
\hline & Até nove anos estudo & 27 & 46,55 & 31 & 53,45 & 2,424 & $1,131-5,193$ & 0,0228 \\
\hline & Acima de nove anos estudo & 38 & 67,86 & 18 & 32,14 & Ref. & & \\
\hline & Bens & & & & & & & \\
\hline & Até oito bens & 39 & 52,70 & 35 & 47,30 & 1,731 & $0,785-3,815$ & 0,1738 \\
\hline & Acima de oito bens & 27 & 65,85 & 14 & 34,15 & Ref. & & \\
\hline & No pessoas residentes & & & & & & & \\
\hline & Até quatro pessoas & 50 & 67,57 & 24 & 32,43 & Ref. & & \\
\hline & Acima de quatro pessoas & 16 & 39,02 & 25 & 60,98 & 3,255 & $1,471-7,202$ & 0,0036 \\
\hline \multirow{3}{*}{$\begin{array}{l}\text { Fatores } \\
\text { facilitadores }\end{array}$} & Renda familiar & & & & & & & \\
\hline & Até R \$ 500,00 & 41 & 51,25 & 39 & 48,75 & 2,537 & $1,049-6,132$ & 0,0387 \\
\hline & Acima de $\mathrm{R} \$ 500,00$ & 24 & 72,73 & 9 & 27,27 & Ref. & & \\
\hline \multirow[t]{3}{*}{ Bloco II } & Serviço de referência & & & & & & & \\
\hline & Público & 53 & 54,64 & 44 & 45,36 & 2,158 & $0,714-6,524$ & 0,1728 \\
\hline & Privado/convênios/ outros & 13 & 72,22 & 5 & 27,78 & Ref. & & \\
\hline \multirow{2}{*}{$\begin{array}{l}\text { Necessidade } \\
\text { percebida }\end{array}$} & Motivo da consulta & & & & & & & \\
\hline & Revisão/prevenção & 21 & 67,74 & 10 & 32,26 & Ref. & & \\
\hline \multirow[t]{10}{*}{ Bloco III } & $\begin{array}{l}\text { Dor/extração/tratamentos/ } \\
\text { outros }\end{array}$ & 45 & 53,57 & 39 & 46,43 & 1,820 & $0,765-4,329$ & 0,1757 \\
\hline & Satisfação com saúde bucal & & & & & & & \\
\hline & Satisfeito & 43 & 71,67 & 17 & 28,33 & Ref. & & \\
\hline & Insatisfeito & 23 & 44,23 & 29 & 55,77 & 3,189 & $1,456-6,986$ & 0,0037 \\
\hline & Vergonha ao sorrir & & & & & & & \\
\hline & Não & 59 & 64,13 & 33 & 35,87 & Ref. & & \\
\hline & $\operatorname{Sim}$ & 7 & 30,43 & 16 & 69,57 & 4,086 & $1,526-10,943$ & 0,0051 \\
\hline & Deixou de dormir & & & & & & & \\
\hline & Não & 58 & 62,37 & 35 & 37,63 & Ref. & & \\
\hline & Sim & 8 & 36,36 & 14 & 63,64 & 2,900 & $1,105-7,609$ & 0,0305 \\
\hline
\end{tabular}

OR: odds ratio; IC: intervalo de confiança; Ref.: nível de referência - o nível de referência da variável dependente foi considerado "em risco".

Fonte: Elaborado pelos autores.

em substituição e ou complemetação ao exame odontológico normativo ${ }^{33}$.

Estar insatisfeito com a saúde bucal e ter vergonha ao sorrir, aspectos relatados como autopercepção pelos sujeitos do estudo, colocam-nos como potencialmente necessitados de tratamento, ao mesmo tempo em que são esses mesmos sujeitos que têm as maiores chance de estar em risco familiar. Isso possibilita que a classificação de vulnera- bilidade/risco como instrumento de acesso para o planejamento das ações voltadas às famílias mais vulneráveis possa direcionar ações de busca ativa.

Uma ação proativa por parte das ESB utilizando a classificação de vulnerabilidade/risco familiar como ordenadora das ações permitiria uma abertura em termos de maior acessibilidade, com equidade e organização, pois podem ser criados espaços nas agendas para as famílias de 
Tabela 2. Modelos 1 e 2 de regressão logística múltipla hierarquizada ajustados para descrever a relação das variáveis independentes com o risco familiar. Ubirajara, São Paulo, 2017.

\begin{tabular}{|c|c|c|c|c|c|c|c|}
\hline & & \multicolumn{3}{|c|}{ Modelo 1} & \multicolumn{3}{|c|}{ Modelo 2} \\
\hline & & OR & IC $95 \%$ & $\begin{array}{c}\text { p- } \\
\text { valor }\end{array}$ & OR & IC $95 \%$ & $\begin{array}{c}\text { p- } \\
\text { valor }\end{array}$ \\
\hline \multirow{3}{*}{$\begin{array}{l}\text { Fatores } \\
\text { predisponentes }\end{array}$} & Idade & & & & & & \\
\hline & Até 36 anos & Ref. & & & & & \\
\hline & Acima de 36 anos & 1,365 & $0,595-3,135$ & 0,462 & & & \\
\hline \multirow[t]{12}{*}{ Bloco I } & Sexo & & & & & & \\
\hline & Masculino & Ref. & & & & & \\
\hline & Feminino & 1,247 & $0,366-4,251$ & 0,723 & & & \\
\hline & Escolaridade & & & & & & \\
\hline & Até nove anos estudo & 1,856 & $0,773-4,455$ & 0,166 & 2,101 & $0,938-4,707$ & 0,071 \\
\hline & Acima de nove anos estudo & Ref. & & & Ref. & & \\
\hline & Bens & & & & & & \\
\hline & Até oito bens & 1,732 & $0,686-4,373$ & 0,244 & & & \\
\hline & Acima de oito bens & Ref. & & & & & \\
\hline & No de pessoas residentes & & & & & & \\
\hline & Até quatro pessoas & Ref. & & & Ref. & & \\
\hline & Acima de quatro pessoas & 3,402 & $1,442-8,023$ & 0,005 & 2,808 & $1,192-6,618$ & 0,018 \\
\hline \multirow{3}{*}{$\begin{array}{l}\text { Fatores } \\
\text { facilitadores }\end{array}$} & Renda & & & & & & \\
\hline & Até R\$ 500,00 & & & & 1,540 & $0,577-4,108$ & 0,388 \\
\hline & Acima de $\mathrm{R} \$ 500,00$ & & & & Ref. & & \\
\hline \multirow[t]{4}{*}{ Bloco II } & Serviço de referência & & & & & & \\
\hline & Público & & & & 1,468 & $0,435-4,953$ & 0,536 \\
\hline & Privado/convênios/outros & & & & Ref. & & \\
\hline & Ajuste do modelo (AICC) & & 153,5917 & & & 149,4133 & \\
\hline
\end{tabular}

OR: odds ratio; IC: intervalo de confiança; Ref. = nível de referência - o nível de referência da variável dependente foi considerado "em risco"; Akaike's information criterion (AICC).

Fonte: Elaborado pelos autores.

maior vulnerabilidade/risco, e por conseguinte seus membros potencialmente mais necessitados teriam a oportunidade de acesso ao serviço ${ }^{17}$.

Uma possível estratégia de busca seriam as visitas domiciliares (VD), por serem atividades utilizadas com a finalidade de subsidiar a intervenção no processo saúde-doença e o planejamento de ações, constituindo-se em um instrumento essencial para operacionalizar parte de um programa ou de uma política por meio do diagnóstico local de saúde de determinado território. Em resumo, as VDs podem efetivar ações, como a busca de famílias de maior vulnerabilidade ${ }^{34,35}$.

Estudos que utilizaram o mesmo instrumento de classificação de vulnerabilidade/risco mostraram que a menor parte das famílias de um território estão em risco, tornando pontual e viável, em termos quantitativos, a busca dessas famílias por intermédio das VDs, uma vez que até 25\% dos espaços nas agendas devem ser destinados às ações que contemplam, entre outras, as próprias VDs, reuniões de equipe e ações coletivas ${ }^{2,9,18}$.
Na perspectiva de ampliação e qualificação das ações em saúde bucal, a organização das VDs se faz necessária, visando a identificação dos riscos e propiciando o acompanhamento e os tratamentos necessários. A visita domiciliar é capaz de criar um vínculo, entendido e adotado como diretriz passível de ser decisiva na interrelação profissional/usuário na atenção à saúde na ESF em busca de novos modos de produzir saúde ${ }^{2,36}$.

Tais ações, como a abertura de espaços nas agendas das equipes de saúde bucal para as famílias mais vulneráveis, juntamente com a estratégia de busca desses usuários por meio das VDs, poderão impactar as agendas das equipes de saúde bucal e torná-las mais equitativas, uma vez que existiriam vagas especificamente reservadas para esses usuários, independentemente da organização local do serviço.

Em pesquisa anterior de validação do mesmo instrumento de classificação de risco utilizado neste estudo (escala de Coelho e Savassi) ${ }^{18}$, os autores evidenciaram que seu uso possibilitou 
Tabela 3. Modelos 3 e 4 de regressão logística múltipla hierarquizada ajustados para descrever a relação das variáveis independentes com o risco familiar. Ubirajara, São Paulo, 2017.

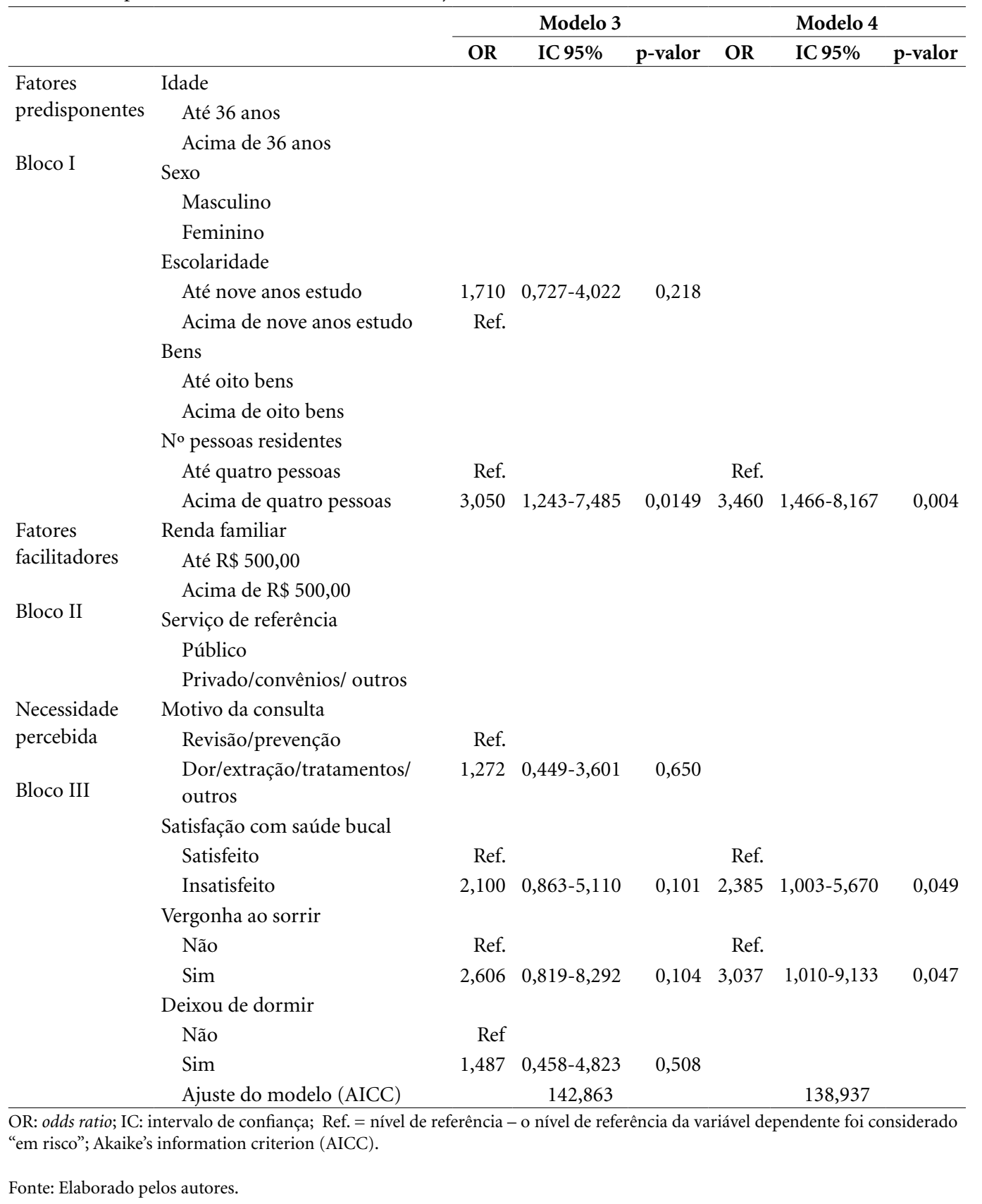

priorizar e estratificar as visitas domiciliares nas áreas de abrangência ${ }^{37}$.

O mesmo instrumento já indicou que em famílias de maior risco ou vulnerabilidade se encontram os indivíduos com maiores chances de apresentarem as duas principais morbidades em saúde bucal: cárie e doença periodontal ${ }^{17,38,39}$, também relacionadas aos indivíduos com pior autopercepção da saúde bucal ${ }^{40}$. Isso corrobora o achado deste estudo, que identificou a auto- percepção da insatisfação com a saúde bucal e a vergonha ao sorrir.

Assim, ao alcançar as famílias com maiores riscos, pode-se alcançar os indivíduos com as mais chances de sofrerem das duas principais morbidades em saúde bucal e com pior autopercepção, mesmo com um número de famílias não tão grande $(\mathrm{n}=115)$, mas significativo e representativo para o estudo, pois foi obtido inicialmente por meio de um censo populacional, o que 


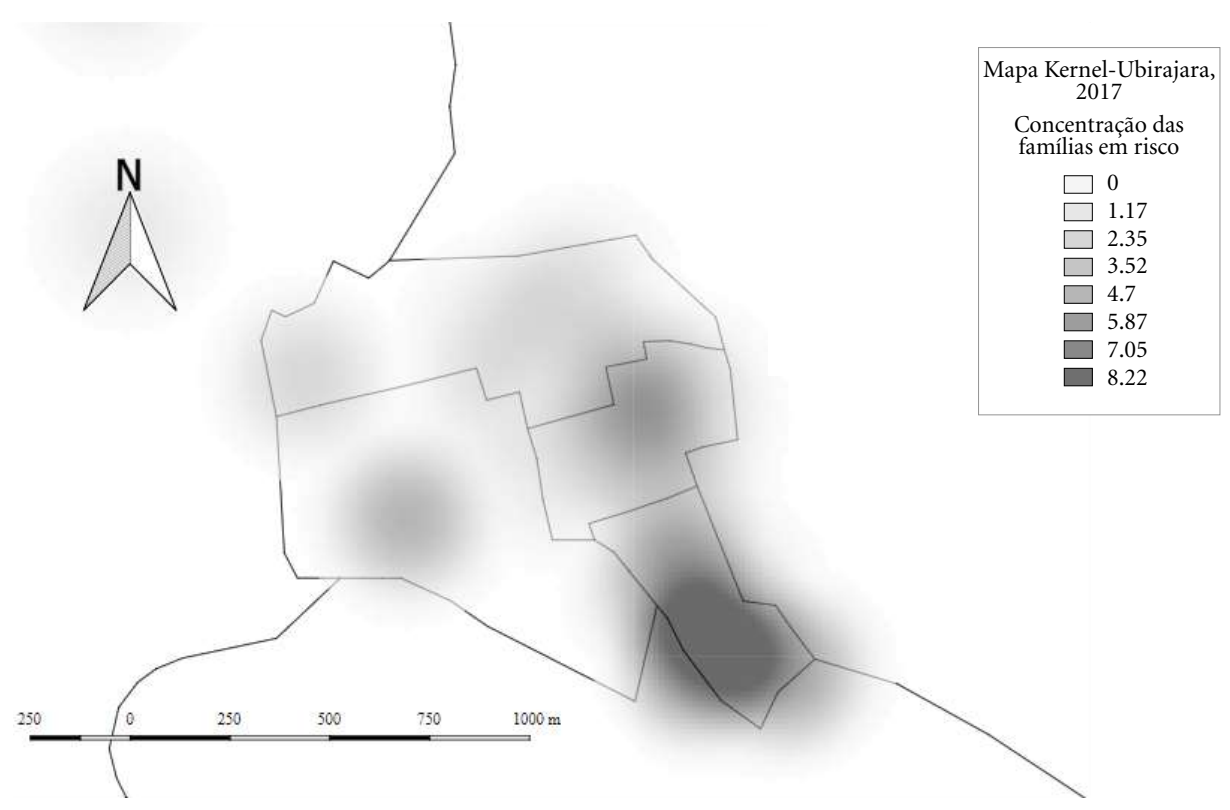

Figura 1. Mapa de Kernel das famílias em risco. Ubirajara, São Paulo, 2017.

Fonte: Elaborado pelos autores.

representa a totalidade das famílias sob responsabilidade das ESB.

Um instrumento de classificação de vulnerabilidade/risco familiar que contemple aspectos socioeconômicos pode ajudar a organizar a demanda, permitindo identificar os grupos mais vulneráveis. $\mathrm{O}$ fato de a menor escolaridade, o maior número de residentes no domicílio e a menor renda terem se associado ao risco familiar neste estudo reafirmam achados anteriores que mostram essas mesmas relações quando utilizado o mesmo instrumento de classificação de risco, que também se relacionam com pior autopercepção em saúde bucal. Isso o torna recomendável, pela abrangência alcançada, quando aspectos relacionados ao processo saúde-doença são considerados em suas determinações sociais ${ }^{40,41}$.

A partir da dinamicidade que a classificação de risco utilizada no estudo assume, pelo em função de utilizar informações ou "sentinelas de risco" passíveis de alterações a qualquer momento - como uma mudança de domicílio, que pode afetar a relação morador/cômodo, ou uma alteração de faixa etária, que também tem potencial para modificar a classificação de risco -, tal método pode proporcionar um melhor funcionamento enquanto equipe multiprofissional, por necessitar de constantes trocas de informações entre os membros das equipes acerca das famílias sob suas responsabilidades. As "sentinelas de risco" contidas e concebidas na antiga Ficha A do Sistema de informação da Atenção Básica (SIAB) se mantém na atual proposta do MS, o e-SUS AB, garantindo portanto a possibilidade de elaboração da classificação de Coelho e Savassi, utilizada neste estudo ${ }^{42,43}$.

As ESB na ESF proporcionam um aumento nas taxas de uso dos serviços, mas para que as ações e serviços resultem em práticas de fato resolutivas é necessário um conhecimento adequado da realidade de cada localidade ${ }^{2,44}$. Ao ser responsável por uma área geográfica e poder estabelecer um vínculo territorial com as famílias nele adscritas, é importante que se localize espacialmente as mesmas para que, a partir de análises e possíveis áreas de maiores concentrações, as ações se iniciem prioritariamente naquelas onde o risco familiar for maior.

A análise espacial por meio do mapa de densidade de Kernel mostrou claramente uma área principal de maior concentração de famílias "em risco", o que possibilita que as atenções das equipes de saúde se voltem para essa área e que as ações comecem onde a vulnerabilidade é maior e mais concentrada. Portanto, apresenta-se como uma ferramenta que, na prática cotidiana dos 
serviços, proporciona um ponto de partida localizado e orientado, uma vez que é necessário considerar toda a gama de barreiras à acessibilidade que as populações mais vulneráveis enfrentam, como restrições de transporte, tempo, econômicas e de apoio social ${ }^{45}$.

Essa área de maior concentração, que se trata de um conjunto habitacional popular, pode ser explicada pelo fato de a classificação de risco utilizada se valer de informações socioeconômicas como renda familiar. Para se ter acesso a essas habitações é necessário que as famílias comprovem condições econômicas desfavoráveis, além de serem construídas com padrões definidos e quantidade mínima de cômodos, o que pode fazer com que um maior número de indivíduos vivam em menos cômodos, afetando uma das "sentinelas de risco" que pode alterar a classificação de risco final: é a relação morador/cômodo.

Uma possível limitação do estudo foi a utilização de variáveis independentes obtidas por um questionário já utilizado em um levantamento epidemiológico estadual, selecionadas de acordo com a proposta do modelo teórico de Andersen ${ }^{20}$, que vem passando, desde a sua concepção, por mudanças em seus fatores de determinação, a fim de responder da melhor e mais completa maneira possível às suas propostas. Outra possível limitação diz respeito à questão da validação da escala de vulnerabilidade utilizada, uma vez que a mesma teve sua validação fundamentada em uma metodologia chamada de "validade de face" 37 , que foi amplamente utilizada em psicometria mas que atualmente vem sendo questionada.

Concluímos com este estudo que, pela relação estabelecida entre o instrumento de vulnerabilidade e as condições socioeconômicas e odontológicas relatadas pelos usuários, além do auxílio na identificação visual de áreas de maior concentração de grupos mais vulneráveis, o instrumento pode ser utilizado pelas equipes de saúde bucal para a organização e a priorização com maior equidade nas ações em um território delimitado.

\section{Colaboradores}

JP Neto participou da concepção, do desenho, da coleta de dados, interpretação, redação e aprovação versão final da pesquisa. MLR Sousa trabalhou na concepção, no desenho, na interpretação, redação e aprovação da versão final apresentada. KLM Cortellazzi participou da análise, interpretação dos dados e aprovação versão final do trabalho. 


\section{Referências}

1. Brasil. Portaria no 1444 , de 28 de dezembro de 2000. Reorganização das ações de saúde bucal na atenção básica: portaria de incentivos financeiros. Diário Oficial da União 2000; 29 dez.

2. Brasil. Ministério da Saúde (MS). Coordenação Nacional de Saúde Bucal. Diretrizes da política nacional da saúde bucal. Brasília: MS; 2004.

3. Corrêa GT, Celeste RK. Associação entre a cobertura de equipes de saúde bucal na saúde da família e o aumento na produção ambulatorial dos municípios brasileiros, 1999 e 2011. Cad Saude Publica 2015; 31(12):2588-2598.

4. Dever GEA, Champagne F. A epidemiologia na administração dos serviços de saúde. São Paulo: Pioneira; 1988.

5. Cesar CLG, Goldbaum M. Usos de serviços de saúde. In: Cesar CLG, Carandina L, Alves MCGP, Azevedo MB, Goldbaum M. Saúde e condição de vida em São Paulo - inquérito multicêntrico de saúde no Estado de São Paulo: ISA-SP. São Paulo: Faculdade de Saúde Pública da USP; 2005. p. 185-98.

6. Barata RB. Acesso e uso de serviços de saúde: considerações sobre os resultados da Pesquisa de Condições de Vida 2006. São Paulo Perspect 2008; 22(2):19-29.

7. Baldani MH, Brito WH, Lawder JAC, Mendes YBE, Silva FFM, Antunes JLF. Determinantes individuais da utilização de serviços odontológicos por adultos e idosos de baixa renda. Rev Bras Epidemiol 2010; 13(1):150-162.

8. Luiz RR. Epidemiologia e bioestatística em odontologia. São Paulo: Ateneu; 2008.

9. Coelho FLG, Savassi LCM. Aplicação de risco familiar como instrumento de priorização das visitas domiciliares. Rev Brasil Med 2004; 1(2): 19-26.

10. Brasil. Ministério da Saúde (MS). Secretaria de Atenção à Saúde. Departamento de Atenção Básica. Instrutivo do Sistema de Informação da Atenção Básica - SIAB. 2a versão. Brasília: MS; 2011.

11. Lage JL, Kato M.. Aplicação da escala de Coelho em equipe de saúde da família. In: Anais do $8^{\circ}$ Congresso Brasileiro de Medicina de Família e Comunidade. São Paulo, 15 a 18 jun 2006.

12. Lage JL, Hadad SC. Utilização de indicadores no planejamento de ações de saúde em centro urbano. In: Anais do $8^{\circ}$ Congresso Brasileiro de Medicina de Família e Comunidade. São Paulo, 15 a 18 jun 2006.

13. Savassi LCM, Lage JL, Coelho FLG. Sistematização de instrumento de estratificação de risco familiar: escala de risco familiar de Coelho-Savassi. J Manag Prim Health Care 2012; 3(2):179-185.

14. Sousa MF, Hamann EM. Programa Saúde da Família no Brasil: uma agenda incompleta? [artigo na Internet] 2007 Mar. [acessado 2008 Out 02]. Disponível em: http://www.abrasco.org.br/cienciaesaudecoletiva/artigos/artigo_int.php?id_artigo $=441$

15. Medronho RA. Geoprocessamento e saúde: uma nova abordagem do espaço no processo saúde doença. Rio de Janeiro: Fiocruz/CICT/NECT; 1995.

16. Camara G, Monteiro AMV. Geocomputation techniques for spatial analisys: are they relevant to health data? Cad Saude Publica 2001; 17(5):1059-1081.
17. Peres Neto J, Mendes KLC, Wada RS, Sousa MLR. Relação entre classificações de risco utilizadas para organização da demanda em saúde bucal em município de pequeno porte de São Paulo, Brasil. Cien Saude Colet 2017; 22(6):1905-1911.

18. Menezes AHR, Cardelli AAM, Vieira GB, Martins JT, Fernandes MV, Marrero T-L. Classificação do risco familiar segundo Coelho e Savassi - um relato de experiência. Cienc Cuid Saude 2012; 11(1):190-195.

19. São Paulo. Secretaria do Estado da Saúde. SB São Paulo 2015: pesquisa estadual de saúde bucal. Piracicaba; 2014.

20. Andersen RM. Revisiting the behavioral model and access to medical care: does it matter? J Health Soc Behav 1995; 36(1):1-10.

21. SAS Institute Inc., Cary, NC, USA. release 2010 Sep 4.

22. What3words. Sistema de endereçamento universal. Acessado por http://what3words.com/pt-br/sobre/.

23. Alexandro MS, Richarde MS, Caio APA, José JSC. Modelagem geoestatística dos casos de dengue e da variação termopluviométrica em João Pessoa, Brasil. Soc Nat 2015; 27(1):157-169.

24. Bradshaw J. A taxonomy of social need. In: Maclachlan F, editor. Problems and progress in medical care: essays on current research. London: Oxford University Press; 1972. p. 71-82.

25. Ankola A, Balappanavar A, Hebbal M, Kakodkar P, Nagesh L, Sardana V. Questionnaire vs. clinical surveys: the right choice? A cross-sectional comparative study. Indian J Dent Res 2011; 22(3):494-499.

26. Axelsson G, Helgadóttir S. Comparison of oral health data from self-administered questionnaire and clinical examination. Community Dent Oral Epidemiol 1995; 23(6):365-368.

27. Liu H, Maida CA, Spolsky VW, Shen J, Li H, Zhou X, Marcus M. Calibration of self-reported oral health to clinically determined standards. Community Dent Oral Epidemiol 2010 38(6):527-539.

28. Jones JA, Spiro A, Miller DR, Garcia RI, Kressin NR. Need for dental care in older veterans: assessment of patient-based measures. J Am Geriatr Soc 2002; 50(1):163-168.

29. Vale EB, Mendes ACG, Moreira RS. Autopercepção da saúde bucal entre adultos na região Nordeste do Brasil. Rev Saude Publica 2013; 47(Supl. 3):98-108.

30. Nascimento AR, Andrade FB, César CC. Validade e utilidade da autopercepção de necessidade de tratamento odontológico por adultos e idosos. Cad Saude Publica 2015; 31(8):1765-1774.

31. Schutzhold S, Holtfreter B, Schiffner U, Hoffmann T, Kocher T, Micheelis W. Clinical factors and self-perceived oral health. Eur J Oral Sci 2014; 122(2):134-141.

32. Haikal DS, Paula AMB, Martins AMEBL, Moreira NA, Ferreira EF. Autopercepção da saúde bucal e impacto na qualidade de vida do idoso: uma abordagem quanti-qualitativa. Cien Saude Colet 2011; 16(7):3317-3329.

33. Dalazen CE, Bomfim RA, De-Carli AD. Fatores associados à autopercepção da necessidade de tratamento odontológico e de prótese em idosos brasileiros. Cien Saude Colet 2018; 23(3):945-952.

34. Takahashi RF, Oliveira MAC. A visita domiciliária no contexto da saúde da família. In: Brasil IDS. Manual de enfermagem. Brasília: Ministério da Saúde; 2001. p. 43-46. 
35. Pereira MJB, Mishima SM, Fortuna CM, Matumoto S, Teixeira RA, Ferraz CA, Nakao JRS, Melo MRAC, Anselmi ML. Assistência domiciliar: instrumento para potencializar processos de trabalho na assistência e formação. In: Barros AFR, Santana JP, Santos Neto $\mathrm{PM}$, organizadores. Observatório de recursos humanos em saúde no Brasil: estudos e análise v.2. Rio de Janeiro: Fiocruz; 2004. p. 71-80.

36. Jorge MSB, Pinto DM, Quinderé PHD, Pinto AGA, Sousa FSP, Cavalcante CM. Promoção da saúde mental - tecnologias do cuidado: vínculo, acolhimento, co-responsabilização e autonomia. Cien Saude Colet 2011; 16(7):3051-3060.

37. Melo RHV, Vilar RLA, Ferreira AF, Pereira EJS, Carneiro NEA, Freitas NGHB, Diniz Júnior J. Análise de risco familiar na estratégia saúde da família: uma vivência compartilhada entre preceptores, discentes e agente comunitários de saúde. R-BITS 2013; 3(4):5971.

38. Kobayashi HM. Relação entre classificação de risco de cárie dentária e escala de risco familiar [tese]. Piracicaba: Universidade Estadual de Campinas; 2012.

39. Silva RDR, Amaral RC, Sousa MLR. Relação entre risco de cárie individual e risco familiar da doença priorizando atendimentos. Rev Assoc Paul Cir Dent 2013; 67(2):141-145.

40. Vila Nova FA, Ambrosano GMB, Pereira SM, Pereira AC, Meneghin MC. Associação do risco familiar com saúde bucal, qualidade de vida e variáveis socioeconômicas. Rev Bras Med Fam Comunidade 2015; 10(34):1-9.

41. Gabardo MCL, Moysés SJ, Moysés ST, Olandoski M, Olinto MTA, Pattussi MP. Multilevel analysis of self-perception in oral health and associated factors in Southern Brazilian adults: a cross-sectional study. Cad Saude Publica 2015; 31(1):49-59.

42. Ministério da Saúde. Cadastro Domiciliar e Territorial [Internet]. [acessado 2018 Dez 20]. Disponível em: http://189.28.128.100/dab/docs/portaldab/documentos/Cadastro_Domiciliar_e_Territorial.pdf

43. Ministério da Saúde. Cadastro Individual [Internet]. [acessado 2018 Out 15]. Disponível em: http://189.28.128.100/dab/docs/portaldab/documentos/esus/ficha_cadastro_individual_v3_2.pdf

44. Corrêa GT, Celeste RK. Associação entre a cobertura de equipes de saúde bucal na saúde da família e o aumento na produção ambulatorial dos municípios brasileiros, 1999 e 2011. Cad Saude Publica 2015; 31(12): 2588-2598.

45. McLafferty SL. GIS and health care. Annu Rev Public Health 2003; 24:25-42.

Artigo apresentado em 31/01/2019

Aprovado em 18/11/2019

Versão final apresentada em 20/11/2019

Editores-chefes: Romeu Gomes, Antônio Augusto Moura da Silva 
